\title{
EXISTENCE AND GLOBAL STABILITY OF POSITIVE PERIODIC SOLUTIONS OF A DISCRETE PREDATOR-PREY SYSTEM WITH DELAYS
}

\author{
LIN-LIN WANG, WAN-TONG LI, AND PEI-HAO ZHAO
}

Received 13 January 2004

We study the existence and global stability of positive periodic solutions of a periodic discrete predator-prey system with delay and Holling type III functional response. By using the continuation theorem of coincidence degree theory and the method of Lyapunov functional, some sufficient conditions are obtained.

\section{Introduction}

Many realistic problems could be solved on the basis of constructing suitable mathematical models, but it is obvious that a perfect model cannot be achieved because even if we could put all possible factors in a model, the model could never predict ecological catastrophes or mother nature caprice. Therefore, the best we can do is to look for analyzable models that describe as well as possible the reality on populations. From a mathematical point of view, the art of good modelling relies on the following: (i) a sound understanding and appreciation of the biological problem; (ii) a realistic mathematical representation of the important biological phenomena; (iii) finding useful solutions, preferably quantitative; (iv) a biological interpretation of the mathematical results in terms of insights and predictions.

Usually a mathematical model could be described by two types of systems: a continuous system or a discrete one. When the size of the population is rarely small or the population has nonoverlapping generations, we may prefer the discrete models. Among all the mathematical models, the predator-prey systems play a fundamental and crucial role (for more details, we refer to $[3,6]$ ). In general, a predator-prey system may have the form

$$
\begin{aligned}
& x^{\prime}=r x\left(1-\frac{x}{K}\right)-\varphi(x) y, \\
& y^{\prime}=y(\mu \varphi(x)-D)
\end{aligned}
$$

where $\varphi(x)$ is the functional response function. Massive work has been done on this issue. We refer to the monographs $[4,10,18,20]$ for general delayed biological systems and to 
$[2,8,9,11,21,24]$ for investigation on predator-prey systems. Here, $\varphi(x)$ may be different response functions: standard type II and type III response functions (Holling [12]), Ivlev's functional response (Ivlev [17]), and Rosenzweig functional response (Rosenzweig [22]). Systems with Holling-type functional response have been investigated by many authors, see, for example, Hsu and Huang [13], Rosenzweig and MacArthur [22, 23]. They studied the stability of the equilibria, existence of Hopf bifurcation, limit cycles, homoclinic loops, and even catastrophe.

On the other hand, in view of the periodic variation of the environment (e.g., food supplies, mating habits, seasonal affects of weather, etc.), it would be of interest to study the global existence and global stability of positive solutions for periodic systems [18]. Recently, some excellent existence results have been obtained by using the coincidence degree method (see, e.g., [5, 14, 15, 16, 19, 27]).

Motivated by the above considerations, we will consider the discrete predator-prey system with Holling type III functional response. The corresponding continuous system which has been investigated in our previous articles $[25,26]$ with discrete delays takes the form

$$
\begin{aligned}
& N_{1}^{\prime}(t)=N_{1}(t)\left[b_{1}(t)-a_{1}(t) N_{1}\left(t-\tau_{1}\right)\right]-\frac{\alpha_{1}(t) N_{1}^{2}(t) N_{2}(t-\sigma)}{1+m N_{1}^{2}(t)}, \\
& N_{2}^{\prime}(t)=N_{2}(t)\left[-b_{2}(t)-a_{2}(t) N_{2}(t)+\frac{\alpha_{2}(t) N_{1}^{2}\left(t-\tau_{2}\right)}{1+m N_{1}^{2}\left(t-\tau_{2}\right)}\right],
\end{aligned}
$$

where $N_{1}(t)$ and $N_{2}(t)$ represent the densities of the prey population and predator population at time $t$, respectively; $m, \tau_{1}, \tau_{2}$, and $\sigma$ are nonnegative constants; $a_{1}(t), b_{1}(t), \alpha_{1}(t)$, $a_{2}(t), b_{2}(t)$, and $\alpha_{2}(t)$ are all continuous functions; $b_{1}(t)$ stands for prey intrinsic growth rate, $b_{2}(t)$ stands for the death rate of the predator, $m$ stands for half capturing saturation; the function $N_{1}(t)\left[b_{1}(t)-a_{1}(t) N_{1}\left(t-\tau_{1}\right)\right]$ represents the specific growth rate of the prey in the absence of predator; and $N_{1}^{2}(t) /\left(1+m N_{1}^{2}(t)\right)$ denotes the predator response function, which reflects the capture ability of the predator.

We assume that the average growth rates in (1.2) change at regular intervals of time, then we can incorporate this aspect in (1.2) and obtain the following modified system:

$$
\begin{aligned}
& \frac{1}{N_{1}(t)} \frac{d N_{1}(t)}{d t}=\left[b_{1}([t])-a_{1}([t]) N_{1}\left([t]-\left[\tau_{1}\right]\right)\right]-\frac{\alpha_{1}([t]) N_{1}([t]) N_{2}([t]-[\sigma])}{1+m N_{1}^{2}([t])}, \\
& \frac{1}{N_{2}(t)} \frac{d N_{2}(t)}{d t}=-b_{2}([t])-a_{2}([t]) N_{2}([t])+\frac{\alpha_{2}([t]) N_{1}^{2}\left([t]-\left[\tau_{2}\right]\right)}{1+m N_{1}^{2}\left([t]-\left[\tau_{2}\right]\right)}, \quad t \neq 0,1,2, \ldots,
\end{aligned}
$$

where $[t]$ denotes the integer part of $t, t \in(0,+\infty)$. By a solution of (1.3) we mean a function $N=\left(N_{1}, N_{2}\right)^{T}$, which is defined for $t \in(0,+\infty)$, and possesses the following properties:

(1) $N$ is continuous on $[0,+\infty)$;

(2) the derivatives $d N_{1}(t) / d t, d N_{2}(t) / d t$ exist at each point $t \in[0,+\infty)$ with the possible exception of the points $t \in\{0,1,2, \ldots\}$, where left-sided derivatives exist;

(3) the equations in (1.3) are satisfied on each interval $[k, k+1)$ with $k=0,1,2, \ldots$ 
On any interval of the form $[k, k+1), k=0,1,2, \ldots$, we can integrate (1.3) and obtain for $k \leq t<k+1, k=0,1,2, \ldots$,

$$
\begin{aligned}
& N_{1}(t)=N_{1}(k) \exp \left\{\left[b_{1}(k)-a_{1}(k) N_{1}\left(k-\left[\tau_{1}\right]\right)-\frac{\alpha_{1}(k) N_{1}(k) N_{2}(k-[\sigma])}{1+m N_{1}^{2}(k)}\right](t-k)\right\}, \\
& N_{2}(t)=N_{2}(k) \exp \left\{\left[-b_{2}(k)-a_{2}(k) N_{2}(k)+\frac{\alpha_{2}(k) N_{1}^{2}\left(k-\left[\tau_{2}\right]\right)}{1+m N_{1}^{2}\left(k-\left[\tau_{2}\right]\right)}\right](t-k)\right\} .
\end{aligned}
$$

Let $t \rightarrow k+1$; we obtain from (1.4) that

$$
\begin{aligned}
& N_{1}(k+1)=N_{1}(k) \exp \left\{b_{1}(k)-a_{1}(k) N_{1}\left(k-\left[\tau_{1}\right]\right)-\frac{\alpha_{1}(k) N_{1}(k) N_{2}(k-[\sigma])}{1+m N_{1}^{2}(k)}\right\}, \\
& N_{2}(k+1)=N_{2}(k) \exp \left\{-b_{2}(k)-a_{2}(k) N_{2}(k)+\frac{\alpha_{2}(k) N_{1}^{2}\left(k-\left[\tau_{2}\right]\right)}{1+m N_{1}^{2}\left(k-\left[\tau_{2}\right]\right)}\right\},
\end{aligned}
$$

which is a discrete time analogue of system (1.2), where $N_{1}(t), N_{2}(t)$ are the densities of the prey population and predator population at time $t$.

Let $\mathbb{Z}, \mathbb{Z}^{+}, \mathbb{R}, \mathbb{R}^{+}$, and $\mathbb{R}^{2}$ denote the sets of all integers, nonnegative integers, real numbers, nonnegative real numbers, and two-dimensional Euclidean vector space, respectively. Throughout this paper, we always assume that $b_{i}: \mathbb{Z} \rightarrow \mathbb{R}$ and $a_{i}, \alpha_{i}: \mathbb{Z} \rightarrow \mathbb{R}^{+}$ $(i=1,2)$ are periodic functions such that

$$
b_{i}(k+\omega)=b_{i}(k), \quad a_{i}(k+\omega)=a_{i}(k), \quad \alpha_{i}(k+\omega)=\alpha_{i}(k), \quad i=1,2,
$$

for any $k \in \mathbb{Z}$ and $\bar{b}_{i}>0(i=1,2)$, where $\omega$ is a positive integer and $\overline{b_{i}}$ is defined as below.

For convenience, we denote

$$
I_{\omega}=\{0,1, \ldots, \omega-1\}, \quad \bar{g}=\frac{1}{\omega} \sum_{k=0}^{\omega-1} g(k), \quad \bar{G}=\frac{1}{\omega} \sum_{k=0}^{\omega-1}|g(k)|,
$$

where $\{g(k)\}$ is an $\omega$-periodic sequence of real numbers defined for $k \in \mathbb{Z}$.

The exponential form of (1.5) assures that for any initial condition $N(0)>0, N(k)$ remains positive. In the rest of this paper, for biological reasons, we only consider solutions $N(k)$ with

$$
N_{i}(-k) \geq 0, \quad k=1,2, \ldots, \max \left\{\left[\tau_{1}\right],\left[\tau_{2}\right],[\sigma]\right\}, \quad N_{i}(0)>0, \quad i=1,2 .
$$




\section{Existence of positive periodic solution}

In order to obtain the existence of positive periodic solution of (1.5), for the reader's convenience, we will summarize in the following a few concepts and results from [7] that will be basic for this section.

Let $X, \mathbb{Z}$ be normed vector spaces, $L:$ Dom $L \subset X \rightarrow \mathbb{Z}$ a linear mapping, and $N: X \rightarrow \mathbb{Z}$ a continuous mapping. The mapping $L$ will be called a Fredholm mapping of index zero if $\operatorname{dim} \operatorname{Ker} L=\operatorname{Codim} \operatorname{Im} L<+\infty$ and $\operatorname{Im} L$ is closed in $\mathbb{Z}$. If $L$ is a Fredholm mapping of index zero, there exist continuous projections $P: X \rightarrow X$ and $Q: \mathbb{Z} \rightarrow \mathbb{Z}$ such that $\operatorname{Im} P=\operatorname{Ker} L$, $\operatorname{Im} L=\operatorname{Ker} Q=\operatorname{Im}(I-Q)$. It follows that $L \mid \operatorname{Dom} L \cap \operatorname{Ker} P:(I-P) X \rightarrow \operatorname{Im} L$ is invertible. We denote the inverse of the map $L$ by $K_{P}$. If $\Omega$ is an open bounded subset of $X$, the mapping $N$ will be called $L$-compact on $\bar{\Omega}$ if $Q N(\bar{\Omega})$ is bounded and $K_{P}(I-Q) N: \bar{\Omega} \rightarrow X$ is compact. Since $\operatorname{Im} Q$ is isomorphic to $\operatorname{Ker} L$, there exists an isomorphism $J: \operatorname{Im} Q \rightarrow$ Ker $L$.

In the proof of our main theorem, we will use the following result from Gaines and Mawhin [7].

Lemma 2.1 (continuation theorem). Let $L$ be a Fredholm mapping of index zero and let $N$ be L-compact on $\bar{\Omega}$. Suppose that

(a) for each $\lambda \in(0,1)$, every solution $x$ of $L x=\lambda N x$ satisfies $x \notin \partial \Omega$;

(b) $Q N x \neq 0$ for each $x \in \partial \Omega \cap \operatorname{Ker} L$ and

$$
\operatorname{deg}\{J Q N, \Omega \cap \operatorname{Ker} L, 0\} \neq 0 .
$$

Then the operator equation $L x=N x$ has at least one solution lying in $\operatorname{Dom} L \cap \bar{\Omega}$.

Now we state two lemmas which are useful to prove the main theorem for the existence of a positive $\omega$-periodic solution.

Lemma 2.2 (see [5]). Let $g: \mathbb{Z} \rightarrow \mathbb{R}$ be a function satisfying $g(k+\omega)=g(k), k \in \mathbb{Z}$. Then for any fixed $k_{1}, k_{2} \in I_{\omega}$ and $k \in \mathbb{Z}$,

$$
\begin{aligned}
& g(k) \leq g\left(k_{1}\right)+\sum_{k=0}^{\omega-1}|g(k+1)-g(k)|, \\
& g(k) \geq g\left(k_{2}\right)-\sum_{k=0}^{\omega-1}|g(k+1)-g(k)| .
\end{aligned}
$$

Lemma 2.3. If $\left(\mathrm{h}_{1}\right)\left(\bar{\alpha}_{2}-m \bar{b}_{2}\right)^{-1 / 2}\left(\bar{b}_{2}\right)^{1 / 2}<\bar{b}_{1} / \bar{a}_{1} \leq 27 / m^{2}$ and $\left(\mathrm{h}_{2}\right) \bar{\alpha}_{2}>m \bar{b}_{2}$ hold, then the system of algebraic equations

$$
\begin{aligned}
& \bar{b}_{1}-\bar{a}_{1} u_{1}-\bar{\alpha}_{1} \frac{u_{1} u_{2}}{1+m u_{1}^{2}}=0 \\
& \bar{b}_{2}+\bar{a}_{2} u_{2}-\bar{\alpha}_{2} \frac{u_{1}^{2}}{1+m u_{1}^{2}}=0
\end{aligned}
$$

has a unique solution $\left(u_{1}^{*}, u_{2}^{*}\right)^{T} \in \mathbb{R}^{2}$ with $u_{i}^{*}>0, i=1,2$. 
Proof. Consider the functions

$$
\begin{array}{ll}
f\left(u_{1}\right)=\frac{\left(1+m u_{1}^{2}\right)\left(\bar{b}_{1}-\bar{a}_{1} u_{1}\right)}{\bar{\alpha}_{1} u_{1}}, & u_{1}>0, \\
g\left(u_{1}\right)=\frac{-\bar{b}_{2}+\left(\bar{\alpha}_{2}-m \bar{b}_{2}\right) u_{1}^{2}}{\bar{a}_{2}\left(1+m u_{1}^{2}\right)}, & u_{1}>0 .
\end{array}
$$

It is easy to see that

$$
\begin{gathered}
f^{\prime}\left(u_{1}\right)=\frac{1}{\bar{\alpha}_{1}}\left(\frac{-\bar{b}_{1}}{u_{1}^{2}}+m \bar{b}_{1}-2 m \bar{a}_{1} u_{1}\right), \\
f^{\prime \prime}\left(u_{1}\right)=\frac{1}{\bar{\alpha}_{1}}\left(\frac{2 \bar{b}_{1}}{u_{1}^{3}}-2 m \bar{a}_{1}\right) .
\end{gathered}
$$

From $\left(\mathrm{h}_{1}\right)$ we know that

$$
f^{\prime}\left(u_{1}\right) \leq 0 .
$$

Notice that

$$
\begin{gathered}
f(0)=+\infty, \quad f(+\infty)=-\infty, \\
g(0)=\frac{-\bar{b}_{2}}{\bar{a}_{2}}<0, \quad g(+\infty)=\frac{\left(\bar{\alpha}_{2}-m \bar{b}_{2}\right)}{\bar{a}_{2}\left(1+m u_{1}^{2}\right)},
\end{gathered}
$$

and in view of $\left(h_{2}\right)$, we have

$$
g^{\prime}\left(u_{1}\right)>0 \text { for } u_{1}>0 .
$$

From the above discussion we may conclude that the curve $f\left(u_{1}\right)=g\left(u_{1}\right)$ has only a unique zero point. It follows that the algebraic equations (2.3) have a unique solution. The proof is complete.

Define

$$
l_{2}=\left\{y=y(k): y(k) \in \mathbb{R}^{2}, k \in \mathbb{Z}\right\}
$$

For $\theta=\left(\theta_{1}, \theta_{2}\right)^{T} \in \mathbb{R}^{2}$, define $|\theta|=\max \left\{\theta_{1}, \theta_{2}\right\}$. Let $l^{\omega} \subset l_{2}$ denote the subspace of all $\omega$-periodic sequences equipped with the norm

$$
\|y\|=\max _{k \in I_{\omega}}|y(k)|,
$$


that is,

$$
l^{\omega}=\left\{y=y(k): y(k+\omega)=y(k), y(k) \in \mathbb{R}^{2}, k \in \mathbb{Z}\right\}
$$

It is not difficult to show that $l^{\omega}$ is a finite-dimensional Banach space.

Set

$$
\begin{aligned}
& l_{0}^{\omega}=\left\{y=y(k) \in l^{\omega}: \sum_{k=0}^{\omega-1} y(k)=0\right\}, \\
& l_{c}^{\omega}=\left\{y=y(k) \in l^{\omega}: y(k)=h \in \mathbb{R}^{2}, k \in \mathbb{Z}\right\} .
\end{aligned}
$$

Then it follows that $l_{0}^{\omega}$ and $l_{c}^{\omega}$ are both closed linear subspaces of $l^{\omega}$ and

$$
l^{\omega}=l_{0}^{\omega} \oplus l_{c}^{\omega}, \quad \operatorname{dim} l_{c}^{\omega}=2 .
$$

Now we state our main result of this section.

Theorem 2.4. Assume that $\left(\mathrm{h}_{1}\right),\left(\mathrm{h}_{3}\right) 2 \sqrt{m} \bar{b}_{1}>\bar{\alpha}_{1} \exp \left\{H_{21}\right\}$, and $\left(\mathrm{h}_{4}\right)$

$$
\frac{\exp \left\{2 H_{12}\right\}}{1+m \exp \left\{2 H_{12}\right\}} \bar{\alpha}_{2}>\bar{b}_{2}
$$

hold, where

$$
\begin{aligned}
& H_{21}=\ln \left\{\frac{\bar{\alpha}_{2}-m \bar{b}_{2}}{m \bar{a}_{2}}\right\}+\left(\bar{B}_{2}+\bar{b}_{2}\right) \omega, \\
& H_{12}=\ln \left\{\frac{2 \sqrt{m} \bar{b}_{1}-\bar{\alpha}_{1} \exp \left\{H_{21}\right\}}{2 \sqrt{m} \bar{a}_{1}}\right\}-\left(\bar{B}_{1}+\bar{b}_{1}\right) \omega .
\end{aligned}
$$

Then (1.5) has at least one positive w-periodic solution.

Proof. Make the change of variables

$$
N_{1}(t)=\exp \left\{x_{1}(t)\right\}, \quad N_{2}(t)=\exp \left\{x_{2}(t)\right\}
$$


then (1.5) can be reformulated as

$$
\begin{aligned}
x_{1}(k+1)-x_{1}(k)= & b_{1}(k)-a_{1}(k) \exp \left\{x_{1}\left(k-\left[\tau_{1}\right]\right)\right\} \\
& -\frac{\alpha_{1}(k) \exp \left\{x_{1}(k)+x_{2}(k-[\sigma])\right\}}{1+m \exp \left\{2 x_{1}(k)\right\}}, \\
x_{2}(k+1)-x_{2}(k)= & -b_{2}(k)-a_{2}(k) \exp \left\{x_{2}(k)\right\} \\
& +\frac{\alpha_{2}(k) \exp \left\{2 x_{1}\left(k-\left[\tau_{2}\right]\right)\right\}}{1+m \exp \left\{2 x_{1}\left(k-\left[\tau_{2}\right]\right)\right\}} .
\end{aligned}
$$

Define

$$
\begin{aligned}
& X=Y=l^{\omega}, \quad(L x)(k)=x(k+1)-x(k), \\
& (N x)(k)=\left[\begin{array}{c}
b_{1}(k)-a_{1}(k) \exp \left\{x_{1}\left(k-\left[\tau_{1}\right]\right)\right\}-\frac{\alpha_{1}(k) \exp \left\{x_{1}(k)+x_{2}(k-[\sigma])\right\}}{1+m \exp \left\{2 x_{1}(k)\right\}} \\
-b_{2}(k)-a_{2}(k) \exp \left\{x_{2}(k)\right\}+\frac{\alpha_{2}(k) \exp \left\{2 x_{1}\left(k-\left[\tau_{2}\right]\right)\right\}}{1+m \exp \left\{2 x_{1}\left(k-\left[\tau_{2}\right]\right)\right\}}
\end{array}\right] \\
& \equiv\left[\begin{array}{c}
\triangle_{1}(k) \\
\triangle_{2}(k)
\end{array}\right]
\end{aligned}
$$

for any $x \in X$ and $k \in \mathbb{Z}$. It is easy to see that $L$ is a bounded linear operator,

$$
\begin{gathered}
\operatorname{Ker} L=l_{c}^{\omega}, \quad \operatorname{Im} L=l_{0}^{\omega}, \\
\operatorname{dim} \operatorname{Ker} L=2=\operatorname{codim} \operatorname{Im} L ;
\end{gathered}
$$

then it follows that $L$ is a Fredholm mapping of index zero.

Set

$$
\begin{aligned}
& P x=\frac{1}{\omega} \sum_{k=0}^{\omega-1} x(s), \quad x \in X, \\
& Q z=\frac{1}{\omega} \sum_{k=0}^{\omega-1} z(s), \quad z \in Y,
\end{aligned}
$$

and $P, Q$ are continuous projectors such that

$$
\operatorname{Im} P=\operatorname{Ker} L, \quad \operatorname{Ker} Q=\operatorname{Im} L=\operatorname{Im}(I-Q)
$$

Furthermore, the generalized inverse to $L$,

$$
K_{P}: \operatorname{Im} L \longrightarrow \operatorname{Ker} P \cap \operatorname{Dom} L,
$$


exists and can be read as

$$
K_{P}(z)=\sum_{s=0}^{k-1} z(s)-\frac{1}{\omega} \sum_{s=0}^{\omega-1}(\omega-s) z(s) .
$$

Thus,

$$
\begin{aligned}
& Q N x=\left[\begin{array}{c}
\frac{1}{\omega} \sum_{k=0}^{\omega-1}\left[b_{1}(k)-a_{1}(k) \exp \left\{x_{1}\left(k-\left[\tau_{1}\right]\right)\right\}\right. \\
\left.-\frac{\alpha_{1}(k) \exp \left\{x_{1}(k)+x_{2}(k-[\sigma])\right\}}{1+m \exp \left\{2 x_{1}(k)\right\}}\right] \\
\frac{1}{\omega} \sum_{k=0}^{\omega-1}\left[-b_{2}(k)-a_{2}(k) \exp \left\{x_{2}(k)\right\}+\frac{\alpha_{2}(k) \exp \left\{2 x_{1}\left(k-\left[\tau_{2}\right]\right)\right\}}{1+m \exp \left\{2 x_{1}\left(k-\left[\tau_{2}\right]\right)\right\}}\right]
\end{array}\right], \\
& K_{P}(I-Q) N x=\left[\begin{array}{l}
\frac{1}{\omega} \sum_{s=0}^{\omega-1} \triangle_{1}(s) \\
\frac{1}{\omega} \sum_{s=0}^{\omega-1} \triangle_{2}(s)
\end{array}\right]-\left[\begin{array}{l}
\frac{1}{\omega} \sum_{s=0}^{\omega-1}(\omega-s) \triangle_{1}(s) \\
\frac{1}{\omega} \sum_{s=0}^{\omega-1}(\omega-s) \triangle_{2}(s)
\end{array}\right] \\
& -\left[\begin{array}{c}
\left(k-\frac{\omega+1}{2}\right) \frac{1}{\omega} \sum_{s=0}^{\omega-1} \triangle_{1}(s) \\
\left(k-\frac{\omega+1}{2}\right) \frac{1}{\omega} \sum_{s=0}^{\omega-1} \triangle_{2}(s)
\end{array}\right] .
\end{aligned}
$$

Obviously, $Q N$ and $K_{P}(I-Q) N$ are continuous. It is not difficult to show that $\overline{K_{P}(I-Q) N(\bar{\Omega})}$ is compact for any open bounded set $\Omega \subset X$ by using the Arzelà-Ascoli theorem. Moreover, $Q N(\bar{\Omega})$ is clearly bounded. Thus, $N$ is $L$-compact on $\bar{\Omega}$ with any open bounded set $\Omega \subset X$.

Now we reach the position to search for an appropriate open bounded set $\Omega$ for the application of the continuation theorem. Corresponding to the operator equation $L x=$ $\lambda N x, \lambda \in(0,1)$,

$$
\begin{aligned}
& x_{1}(k+1)-x_{1}(k)=\lambda\left[b_{1}(k)-a_{1}(k) \exp \left\{x_{1}\left(k-\left[\tau_{1}\right]\right)\right\}-\frac{\alpha_{1}(k) \exp \left\{x_{1}(k)+x_{2}(k-[\sigma])\right\}}{1+m \exp \left\{2 x_{1}(k)\right\}}\right], \\
& x_{2}(k+1)-x_{2}(k)=\lambda\left[-b_{2}(k)-a_{2}(k) \exp \left\{x_{2}(k)\right\}+\frac{\alpha_{2}(k) \exp \left\{2 x_{1}\left(k-\left[\tau_{2}\right]\right)\right\}}{\left.1+m \exp \left\{2 x_{1}\left(k-\left[\tau_{2}\right]\right)\right\}\right] .}\right.
\end{aligned}
$$


Assume that $x(t) \in X$ is an $\omega$-periodic solution of (2.25) for a certain $\lambda \in(0,1)$. Summing on both sides of (2.25) from 0 to $\omega-1$ with respect to $k$, we obtain

$$
\begin{aligned}
& \sum_{k=0}^{\omega-1}\left[x_{1}(k+1)-x_{1}(k)\right] \\
& =\lambda \sum_{k=0}^{\omega-1}\left[b_{1}(k)-a_{1}(k) \exp \left\{x_{1}\left(k-\left[\tau_{1}\right]\right)\right\}-\frac{\alpha_{1}(k) \exp \left\{x_{1}(k)+x_{2}(k-[\sigma])\right\}}{1+m \exp \left\{2 x_{1}(k)\right\}}\right], \\
& \sum_{k=0}^{\omega-1}\left[x_{2}(k+1)-x_{2}(k)\right] \\
& =\lambda \sum_{k=0}^{\omega-1}\left[-b_{2}(k)-a_{2}(k) \exp \left\{x_{2}(k)\right\}+\frac{\alpha_{2}(k) \exp \left\{2 x_{1}\left(k-\left[\tau_{2}\right]\right)\right\}}{1+m \exp \left\{2 x_{1}\left(k-\left[\tau_{2}\right]\right)\right\}}\right] .
\end{aligned}
$$

Notice that

$$
\sum_{k=0}^{\omega-1}\left[x_{1}(k+1)-x_{1}(k)\right]=\sum_{k=0}^{\omega-1}\left[x_{2}(k+1)-x_{2}(k)\right]=0 .
$$

Thus

$$
\begin{aligned}
& \bar{b}_{1} \omega=\sum_{k=0}^{\omega-1}\left[a_{1}(k) \exp \left\{x_{1}\left(k-\left[\tau_{1}\right]\right)\right\}+\frac{\alpha_{1}(k) \exp \left\{x_{1}(k)+x_{2}(k-[\sigma])\right\}}{1+m \exp \left\{2 x_{1}(k)\right\}}\right], \\
& \bar{b}_{2} \omega=\sum_{k=0}^{\omega-1}\left[-a_{2}(k) \exp \left\{x_{2}(k)\right\}+\frac{\alpha_{2}(k) \exp \left\{2 x_{1}\left(k-\left[\tau_{2}\right]\right)\right\}}{1+m \exp \left\{2 x_{1}\left(k-\left[\tau_{2}\right]\right)\right\}}\right] .
\end{aligned}
$$

From (2.25), (2.28), and (2.29), we obtain

$$
\begin{aligned}
& \sum_{k=0}^{\omega-1}\left|x_{1}(k+1)-x_{1}(k)\right| \\
& \quad \leq \sum_{k=0}^{\omega-1}\left[\left|b_{1}(k)\right|+a_{1}(k) \exp \left\{x_{1}\left(k-\left[\tau_{1}\right]\right)\right\}+\frac{\alpha_{1}(k) \exp \left\{x_{1}(k)+x_{2}(k-[\sigma])\right\}}{1+m \exp \left\{2 x_{1}(k)\right\}}\right] \\
& \quad=\left(\bar{B}_{1}+\bar{b}_{1}\right) \omega
\end{aligned}
$$

$$
\begin{aligned}
& \sum_{k=0}^{\omega-1}\left|x_{2}(k+1)-x_{2}(k)\right| \\
& \quad \leq \sum_{k=0}^{\omega-1}\left|b_{2}(k)\right|+\sum_{k=0}^{\omega-1}\left[-a_{2}(k) \exp \left\{x_{2}(k)\right\}+\frac{\alpha_{2}(k) \exp \left\{2 x_{1}\left(k-\left[\tau_{2}\right]\right)\right\}}{1+m \exp \left\{2 x_{1}\left(k-\left[\tau_{2}\right]\right)\right\}}\right] \\
& \quad=\left(\bar{B}_{2}+\bar{b}_{2}\right) \omega .
\end{aligned}
$$


Note that $x(t)=\left(x_{1}(t), x_{2}(t)\right)^{T} \in X$; then there exist $\xi_{i}, \eta_{i} \in I_{\omega}(i=1,2)$ such that

$$
x_{i}\left(\xi_{i}\right)=\min _{k \in I_{\omega}} x_{i}(k), \quad x_{i}\left(\eta_{i}\right)=\max _{k \in I_{\omega}} x_{i}(k), \quad i=1,2 .
$$

In view of (2.29), we get

$$
\bar{b}_{2}+\bar{a}_{2} \exp \left\{x_{2}\left(\xi_{2}\right)\right\} \leq \bar{\alpha}_{2} \frac{\exp \left\{2 x_{1}\left(k-\left[\tau_{2}\right]\right)\right\}}{1+m \exp \left\{2 x_{1}\left(k-\left[\tau_{2}\right]\right)\right\}} \leq \frac{\bar{\alpha}_{2}}{m},
$$

thus

$$
x_{2}\left(\xi_{2}\right) \leq \ln \left\{\frac{\bar{\alpha}_{2} / m-\bar{b}_{2}}{\bar{a}_{2}}\right\} .
$$

Therefore, by Lemma 2.2, we obtain

$$
\begin{aligned}
x_{2}(k) & \leq x_{2}\left(\xi_{2}\right)+\sum_{k=0}^{\omega-1}\left|x_{2}(s+1)-x_{2}(s)\right| \\
& \leq \ln \left\{\frac{\bar{\alpha}_{2} / m-\bar{b}_{2}}{\bar{a}_{2}}\right\}+\left(\bar{B}_{2}+\bar{b}_{2}\right) \omega=H_{21} .
\end{aligned}
$$

From (2.28), we know that

$$
\bar{a}_{1} \omega \exp \left\{x_{1}\left(\xi_{1}\right)\right\} \leq \sum_{k=0}^{\omega-1}\left[a_{1}(k) \exp \left\{x_{1}\left(k-\left[\tau_{1}\right]\right)\right\}\right] \leq \bar{b}_{1} \omega,
$$

so we get

$$
x_{1}\left(\xi_{1}\right) \leq \ln \left\{\frac{\bar{b}_{1}}{\bar{a}_{1}}\right\} .
$$

Combine (2.37) with (2.30); also, in view of Lemma 2.2, we conclude that

$$
x_{1}(k) \leq x_{1}\left(\xi_{1}\right)+\sum_{k=0}^{\omega-1}\left|x_{1}(s+1)-x_{1}(s)\right| \leq \ln \left\{\frac{\bar{b}_{1}}{\bar{a}_{1}}\right\}+\left(\bar{B}_{1}+\bar{b}_{1}\right) \omega:=H_{11} .
$$

Formulas (2.35) and (2.28) imply that

$$
\begin{aligned}
\bar{b}_{1} \omega & \leq \sum_{k=0}^{\omega-1}\left[a_{1}(k) \exp \left\{x_{1}\left(\eta_{1}\right)\right\}+\frac{\alpha_{1}(k) \exp \left\{x_{1}(k)\right\} \exp \left\{H_{21}\right\}}{1+m \exp \left\{2 x_{1}(k)\right\}}\right] \\
& \leq \bar{a}_{1} \omega \exp \left\{x_{1}\left(\eta_{1}\right)\right\}+\frac{\bar{\alpha}_{1} \omega \exp \left\{H_{21}\right\}}{2 \sqrt{m}} .
\end{aligned}
$$

Direct calculation yields

$$
x_{1}\left(\eta_{1}\right) \geq \ln \left\{\frac{2 \sqrt{m} \bar{b}_{1}-\bar{\alpha}_{1} \exp \left\{H_{21}\right\}}{2 \sqrt{m} \bar{a}_{1}}\right\},
$$


thus, by Lemma 2.2,

$$
\begin{aligned}
x_{1}(k) & \geq x_{1}\left(\eta_{1}\right)-\sum_{k=0}^{\omega-1}\left|x_{1}(s+1)-x_{1}(s)\right| \\
& \geq \ln \left\{\frac{2 \sqrt{m} \bar{b}_{1}-\bar{\alpha}_{1} \exp \left\{H_{21}\right\}}{2 \sqrt{m} \bar{a}_{1}}\right\}-\left(\bar{B}_{1}+\bar{b}_{1}\right) \omega=H_{12} .
\end{aligned}
$$

From (2.29), (2.41), and the monotonicity of the function

$$
\frac{\exp \{2 u\}}{1+m \exp \{2 u\}} \quad(m>0),
$$

we have

$$
\bar{b}_{2} \omega+\bar{a}_{2} \omega \exp \left\{x_{2}\left(\eta_{2}\right)\right\} \geq \sum_{k=0}^{\omega-1} \frac{\alpha_{2}(k) \exp \left\{2 x_{1}\left(\xi_{1}\right)\right\}}{1+m \exp \left\{2 x_{1}\left(\xi_{1}\right)\right\}} \geq \frac{\exp \left\{2 H_{12}\right\}}{1+m \exp \left\{2 H_{12}\right\}} \bar{\alpha}_{2} \omega ;
$$

this means that

$$
x_{2}\left(\eta_{2}\right) \geq \ln \left\{\frac{\left(\exp \left\{2 H_{12}\right\} /\left(1+m \exp \left\{2 H_{12}\right\}\right)\right) \bar{\alpha}_{2}-\bar{b}_{2}}{\bar{a}_{2}}\right\} .
$$

From (2.44), (2.31), and Lemma 2.2, we know that

$$
\begin{aligned}
x_{2}(k) & \geq x_{2}\left(\eta_{2}\right)-\sum_{k=0}^{\omega-1}\left|x_{2}(s+1)-x_{2}(s)\right| \\
& \geq \ln \left\{\frac{\left(\exp \left\{2 H_{12}\right\} /\left(1+m \exp \left\{2 H_{12}\right\}\right)\right) \bar{\alpha}_{2}-\bar{b}_{2}}{\bar{a}_{2}}\right\}-\left(\bar{B}_{2}+\bar{b}_{2}\right) \omega:=H_{22} .
\end{aligned}
$$

Inequalities (2.38) and (2.41) imply that

$$
\left|x_{1}(k)\right| \leq \max \left\{\left|H_{11}\right|,\left|H_{12}\right|\right\}:=H_{1} .
$$

On the other hand, (2.35) and (2.45) lead to

$$
\left|x_{2}(k)\right| \leq \max \left\{\left|H_{21}\right|,\left|H_{22}\right|\right\}:=H_{2} .
$$

Obviously, $H_{1}$ and $H_{2}$ are independent of the choice of $\lambda$. Under the assumptions in Theorem 2.4, by Lemma 2.3, we can easily know that the algebraic equations

$$
\begin{aligned}
& \bar{b}_{1}-\bar{a}_{1} u_{1}-\bar{\alpha}_{1} \frac{u_{1} u_{2}}{1+m u_{1}^{2}}=0 \\
& \bar{b}_{2}+\bar{a}_{2} u_{2}-\bar{\alpha}_{2} \frac{u_{1}^{2}}{1+m u_{1}^{2}}=0
\end{aligned}
$$

have a unique solution $\left(u_{1}^{*}, u_{2}^{*}\right)^{T}$ with $u_{i}^{*}>0(i=1,2)$. 
Let $H=H_{1}+H_{2}+H_{3}$, where $H_{3}>0$ is large enough such that

$$
\left\|\left(\ln \left\{u_{1}^{*}\right\}, \ln \left\{u_{2}^{*}\right\}\right)^{T}\right\|=\max \left\{\left|\ln \left\{u_{1}^{*}\right\}\right|,\left|\ln \left\{u_{2}^{*}\right\}\right|\right\}<H_{3}
$$

and define

$$
\Omega=\left\{x(t)=\left(x_{1}(t), x_{2}(t)\right)^{T} \in X:\|x\|<H\right\}
$$

It is easy to see that $\Omega$ satisfies Lemma 2.1(a). When $x \in \partial \Omega \cap \operatorname{Ker} L=\partial \Omega \cap \mathbb{R}^{2}, x$ is a constant vector in $\mathbb{R}^{2}$ with $\|x\|=H$. Then

$$
Q N x=\left[\begin{array}{c}
\bar{b}_{1}-\bar{a}_{1} \exp \left\{x_{1}\right\}-\bar{\alpha}_{1} \frac{\exp \left\{x_{1}+x_{2}\right\}}{1+m \exp \left\{2 x_{1}\right\}} \\
-\bar{b}_{2}-\bar{a}_{2} \exp \left\{x_{2}\right\}+\bar{\alpha}_{2} \frac{\exp \left\{2 x_{1}\right\}}{1+m \exp \left\{2 x_{1}\right\}}
\end{array}\right] \neq 0 .
$$

Since $\operatorname{Im} P=\operatorname{Ker} L, J$ can be chosen as the identity mapping. In view of the assumptions in Theorem 2.4, direct calculation yields

$$
\operatorname{deg}\{J Q N, \Omega \cap \operatorname{Ker} L, 0\} \neq 0 \text {. }
$$

By now, we have proved that $\Omega$ satisfies all the conditions in Lemma 2.1. Hence (2.17) has at least one solution $\left(x_{1}^{*}(t), x_{2}^{*}(t)\right)^{T}$ in $\operatorname{Dom} L \cap \bar{\Omega}$. Set $N_{1}^{*}(t)=\exp \left\{x_{1}^{*}(t)\right\}, N_{2}^{*}(t)=$ $\exp \left\{x_{2}^{*}(t)\right\}$; then $N^{*}(t)=\left(N_{1}^{*}(t), N_{2}^{*}(t)\right)^{T}$ is a positive $\omega$-periodic solution of (1.5). This completes the proof.

\section{Global asymptotic stability}

The purpose of this section is to present sufficient conditions for the global asymptotic stability of system (1.5) when the delays are all zero. The method we use here is to construct a suitable Lyapunov function.

Theorem 3.1. Assume that $\left(\mathrm{h}_{1}\right),\left(\mathrm{h}_{2}\right)$, and $\left(\mathrm{h}_{3}\right)$ hold and, furthermore, suppose that there exist positive numbers $v, c_{1}$, and $c_{2}$ such that

$$
\begin{gathered}
c_{1} a_{1}(k)+\frac{c_{1} \alpha_{1}(k) \exp \left\{H_{22}\right\}}{1+m \exp \left\{2 H_{11}\right\}}-\frac{c_{1} \alpha_{1}(k) \exp \left\{H_{21}\right\}}{4}-\frac{c_{2} \alpha_{2}(k)}{\sqrt{m}\left(1+m \exp \left\{2 H_{12}\right\}\right)} \geq v, \\
c_{2} a_{2}(k)-c_{1} \frac{\alpha_{1}(k)}{2 \sqrt{m}} \geq v, \\
a_{2}(k) \exp \left\{H_{21}\right\} \leq 1, \\
a_{1}(k) \exp \left\{H_{11}\right\}+\frac{\alpha_{1}(k) \exp \left\{H_{21}\right\}}{2 \sqrt{m}\left(1+m \exp \left\{2 H_{12}\right\}\right)}-\frac{m \alpha_{1}(k) \exp \left\{3 H_{12}+H_{22}\right\}}{\left(1+m \exp \left\{2 H_{11}\right\}\right)^{2}} \leq 1 .
\end{gathered}
$$

Then the positive solution of system (1.5) is globally asymptotically stable. 
Proof. Let $\left\{N_{i}^{*}(k)\right\}(i=1,2)$ be a positive solution of system (1.5). Introduce the change of variables

$$
u_{1}(k)=N_{1}(k)-N_{1}^{*}(k), \quad u_{2}(k)=N_{2}(k)-N_{2}^{*}(k) .
$$

Then, from system (1.5), we can obtain

$$
\begin{aligned}
& u_{1}(k+1)=N_{1}(k) \exp \left\{b_{1}(k)-a_{1}(k) N_{1}(k)-\frac{\alpha_{1}(k) N_{1}(k) N_{2}(k)}{1+m N_{1}^{2}(k)}\right\} \\
& -N_{1}^{*}(k) \exp \left\{b_{1}(k)-a_{1}(k) N_{1}^{*}(k)-\frac{\alpha_{1}(k) N_{1}^{*}(k) N_{2}^{*}(k)}{1+m N_{1}^{* 2}(k)}\right\} \\
& =\left[N_{1}(k) \exp \left\{-a_{1}(k) u_{1}(k)-\alpha_{1}(k)\left[\frac{N_{1}(k) N_{2}(k)}{1+m N_{1}^{2}(k)}-\frac{N_{1}^{*}(k) N_{2}^{*}(k)}{1+m N_{1}^{* 2}(k)}\right]\right\}-N_{1}^{*}(k)\right] \\
& \times \exp \left\{b_{1}(k)-a_{1}(k) N_{1}^{*}(k)-\frac{\alpha_{1}(k) N_{1}^{*}(k) N_{2}^{*}(k)}{1+m N_{1}^{* 2}(k)}\right\} \\
& =\left\{\left[1-a_{1}(k) N_{1}^{*}(k)-\frac{\alpha_{1}(k) N_{1}^{*}(k) N_{2}^{*}(k)\left(1-m N_{1}^{* 2}(k)\right)}{\left(1+m N_{1}^{* 2}(k)\right)^{2}}\right] \frac{u_{1}(k)}{N_{1}^{*}(k)}\right. \\
& \left.-\frac{\alpha_{1}(k) N_{1}^{*}(k)}{1+m N_{1}^{* 2}(k)} u_{2}(k)+f_{1}\right\} N_{1}^{*}(k+1), \\
& u_{2}(k+1)=N_{2}(k) \exp \left\{-b_{2}(k)-a_{2}(k) N_{2}(k)+\frac{\alpha_{2}(k) N_{1}^{2}(k)}{1+m N_{1}^{2}(k)}\right\} \\
& -N_{2}^{*}(k) \exp \left\{-b_{2}(k)-a_{2}(k) N_{2}^{*}(k)+\frac{\alpha_{2}(k) N_{1}^{* 2}(k)}{1+m N_{1}^{* 2}(k)}\right\} \\
& =\left[N_{2}(k) \exp \left\{-a_{2}(k) u_{2}(k)+\frac{\alpha_{2}(k) N_{1}^{2}(k)}{1+m N_{1}^{2}(k)}-\frac{\alpha_{2}(k) N_{1}^{* 2}(k)}{1+m N_{1}^{* 2}(k)}\right\}-N_{2}^{*}(k)\right] \\
& \times \exp \left\{-b_{2}(k)-a_{2}(k) N_{2}^{*}(k)+\frac{\alpha_{2}(k) N_{1}^{* 2}(k)}{1+m N_{1}^{* 2}(k)}\right\} \\
& =\left[\left(1-a_{2}(k) N_{2}^{*}(k)\right) \frac{u_{2}(k)}{N_{2}^{*}(k)}+\frac{2 \alpha_{2}(k) N_{1}^{*}(k)}{\left(1+m N_{1}^{* 2}(k)\right)^{2}} u_{1}(k)+f_{2}\right] \\
& \times N_{2}^{*}(k+1),
\end{aligned}
$$

where $\left|f_{i}\right| /\|u\|$ converges, uniformly with respect to $k \in N$, to zero as $\|u\| \rightarrow 0$.

Define a function $V$ by

$$
V(u(k))=c_{1}\left|\frac{u_{1}(k)}{N_{1}^{*}(k)}\right|+c_{2}\left|\frac{u_{2}(k)}{N_{2}^{*}(k)}\right|
$$


where $c_{1}, c_{2}$ are positive constants given in (3.1). Calculating the difference of $V$ along the solution of the system, we obtain

$$
\begin{aligned}
& \Delta V=c_{1}\left(\left|\frac{u_{1}(k+1)}{N_{1}^{*}(k+1)}\right|-\left|\frac{u_{1}(k)}{N_{1}^{*}(k)}\right|\right)+c_{2}\left(\left|\frac{u_{2}(k+1)}{N_{2}^{*}(k+1)}\right|-\left|\frac{u_{2}(k)}{N_{2}^{*}(k)}\right|\right) \\
& \leq-\left\{c_{1} a_{1}(k)+\frac{c_{1} \alpha_{1}(k) N_{2}^{*}(k)\left(1-m N_{1}^{* 2}(k)\right)}{\left(1+m N_{1}^{* 2}(k)\right)^{2}}\right\}\left|u_{1}(k)\right| \\
& +c_{1} \frac{\alpha_{1}(k) N_{1}^{*}(k)}{1+m N_{1}^{* 2}(k)}\left|u_{2}(k)\right|-c_{2} a_{2}(k)\left|u_{2}(k)\right| \\
& +c_{2} \frac{2 \alpha_{2}(k) N_{1}^{*}(k)}{\left(1+m N_{1}^{* 2}(k)\right)^{2}}\left|u_{1}(k)\right|+\sum_{i=1}^{2} c_{i}\left|f_{i}\right| \\
& =-\left\{c_{1} a_{1}(k)+\frac{c_{1} \alpha_{1}(k) N_{2}^{*}(k)\left(1-m N_{1}^{* 2}(k)\right)}{\left(1+m N_{1}^{* 2}(k)\right)^{2}}-c_{2} \frac{2 \alpha_{2}(k) N_{1}^{*}(k)}{\left(1+m N_{1}^{* 2}(k)\right)^{2}}\right\}\left|u_{1}(k)\right| \\
& -\left\{c_{2} a_{2}(k)-c_{1} \frac{\alpha_{1}(k) N_{1}^{*}(k)}{1+m N_{1}^{* 2}(k)}\right\}\left|u_{2}(k)\right|+\sum_{i=1}^{2} c_{i}\left|f_{i}\right| \\
& \leq-\left\{c_{1} a_{1}(k)+\frac{c_{1} \alpha_{1}(k) \exp \left\{H_{22}\right\}}{1+m \exp \left\{2 H_{11}\right\}}-\frac{c_{1} \alpha_{1}(k) \exp \left\{H_{21}\right\}}{4}\right. \\
& \left.-\frac{c_{2} \alpha_{2}(k)}{\sqrt{m}\left(1+m \exp \left\{2 H_{12}\right\}\right)}\right\}\left|u_{1}(k)\right| \\
& -\left\{c_{2} a_{2}(k)-c_{1} \frac{\alpha_{1}(k)}{2 \sqrt{m}}\right\}\left|u_{2}(k)\right|+\sum_{i=1}^{2} c_{i}\left|f_{i}\right| \text {. }
\end{aligned}
$$

Since $\left|f_{i}\right| /\|u\|$ converges uniformly to zero as $\|u\| \rightarrow 0$, it follows from conditions (3.1) and (3.2) that there is a positive $\sigma$ such that if $k$ is sufficiently large and $\|u\|<\sigma$, then

$$
\Delta V \leq-\frac{v}{2}\left\{\left|u_{1}(k)\right|+\left|u_{2}(k)\right|\right\}<-\frac{v}{4}\|u\| .
$$

This means that the trivial solution of (3.6) is uniformly asymptotically stable, and so is the solution $N^{*}(k)=\left(N_{1}^{*}(k), N_{2}^{*}(k)\right)$ of $(1.5)$.

Notice that

$$
\max \{p(x), q(x)\}=\frac{(|p(x)-q(x)|+p(x)+q(x))}{2} \leq|p(x)|+|q(x)| .
$$

Define

$$
\begin{aligned}
& \Phi(x)=\frac{2 x}{\min \left\{\exp \left\{H_{12}\right\}, \exp \left\{H_{22}\right\}\right\}}, \\
& \Psi(x)=\frac{x}{\max \left\{\exp \left\{H_{11}\right\}, \exp \left\{H_{21}\right\}\right\}} .
\end{aligned}
$$


Then

$$
\Psi(\|u\|) \leq V(u(k)) \leq \Phi(\|u\|)
$$

From the Lyapunov asymptotic stability theorem [1], also in view of the positive definition of $V$ and (3.9), we obtain that the trivial solution of (3.6) is globally asymptotically stable. By the medium of (3.5), we reach the conclusion that the solution $N^{*}(k)=$ $\left(N_{1}^{*}(k), N_{2}^{*}(k)\right)$ of $(1.5)$ is globally asymptotically stable. The proof is complete.

\section{Acknowledgment}

This research was supported by the NNSF of China (10171040), the NSF of Gansu province of China (ZS011-A25-007-Z), the Teaching and Research Award Program for Outstanding Young Teachers in Higher Education Institutions of the Ministry of Education of China, Liu Hui Center for Applied Mathematics of Nankai University, and Tianjin University in China.

\section{References}

[1] R. P. Agarwal, Difference Equations and Inequalities. Theory, Methods, and Applications, Monographs and Textbooks in Pure and Applied Mathematics, vol. 155, Marcel Dekker, New York, 1992.

[2] E. Beretta and Y. Kuang, Convergence results in a well-known delayed predator-prey system, J. Math. Anal. Appl. 204 (1996), no. 3, 840-853.

[3] A. A. Berryman, The origins and evolution of predator-prey theory, Ecology 75 (1992), 15301535.

[4] J. M. Cushing, Integrodifferential Equations and Delay Models in Population Dynamics, Lecture Notes in Biomathematics, vol. 20, Springer-Verlag, Heidelberg, 1977.

[5] M. Fan and K. Wang, Periodic solutions of a discrete time nonautonomous ratio-dependent predator-prey system, Math. Comput. Modelling 35 (2002), no. 9-10, 951-961.

[6] H. I. Freedman, Deterministic Mathematical Models in Population Ecology, Monographs and Textbooks in Pure and Applied Mathematics, vol. 57, Marcel Dekker, New York, 1980.

[7] R. E. Gaines and J. L. Mawhin, Coincidence Degree, and Nonlinear Differential Equations, Lecture Notes in Mathematics, vol. 568, Springer-Verlag, Berlin, 1977.

[8] K. Gopalsamy, Harmless delays in model systems, Bull. Math. Biol. 45 (1983), no. 3, 295-309.

[9] Delayed responses and stability in two-species systems, J. Austral. Math. Soc. Ser. B 25 (1984), no. 4, 473-500.

[10] Stability and Oscillations in Delay Differential Equations of Population Dynamics, Mathematics and Its Applications, vol. 74, Kluwer Academic Publishers, Dordrecht, 1992.

[11] A. Hastings, Delays in recruitment at different trophic levels: effects on stability, J. Math. Biol. 21 (1984), no. 1, 35-44.

[12] C. S. Holling, The functional response of predators to prey density and its role in mimicry and population regulation, Mem. Ent. Sec. Can. (1965), no. 45, 3-60.

[13] S. B. Hsu and T. W. Huang, Global stability for a class of predator-prey systems, SIAM J. Appl. Math. 55 (1995), no. 3, 763-783.

[14] H.-F. Huo and W.-T. Li, Periodic solutions of a periodic two-species competition model with delays, Int. J. Appl. Math. 12 (2003), no. 1, 13-21.

[15] - Periodic solutions of a ratio-dependent food chain model with delays, Taiwanese J. Math. 8 (2004), no. 2, 211-222. 
[16] H.-F. Huo, W.-T. Li, and S. S. Cheng, Periodic solutions of two-species diffusion models with continuous time delays, Demonstratio Math. 35 (2002), no. 2, 433-446.

[17] V. S. Ivlev, Experimental Ecology of the Feeding of Fishes, Yale University Press, Connecticut, 1961.

[18] Y. Kuang, Delay Differential Equations with Applications in Population Dynamics, Mathematics in Science and Engineering, vol. 191, Academic Press, Massachusetts, 1993.

[19] Y. K. Li, Periodic solutions of a periodic delay predator-prey system, Proc. Amer. Math. Soc. 127 (1999), no. 5, 1331-1335.

[20] N. MacDonald, Time Lags in Biological Models, Lecture Notes in Biomathematics, vol. 27, Springer-Verlag, Heidelberg, 1978.

[21] R. M. May, Time delay versus stability in population models with two and three trophic levels, Ecology 54 (1973), no. 2, 315-325.

[22] M. L. Rosenzweig, Paradox of enrichment: destabilization of exploitation ecosystem in ecological time, Science 171 (1971), 385-387.

[23] M. L. Rosenzweig and R. MacArthur, Graphical representation and stability conditions of predator-prey interactions, Amer. Natur. 97 (1963), 209-223.

[24] S. Ruan, Absolute stability, conditional stability and bifurcation in Kolmogorov-type predator-prey systems with discrete delays, Quart. Appl. Math. 59 (2001), no. 1, 159-173.

[25] L.-L. Wang and W.-T. Li, Existence of periodic solutions of a delayed predator-prey system with functional response, Int. J. Math. Sci. 1 (2002), no. 1-2, 55-63.

[26] Existence and global stability of positive periodic solutions of a predator-prey system with delays, Appl. Math. Comput. 146 (2003), no. 1, 167-185.

[27] - Periodic solutions and permanence for a delayed nonautonomous ratio-dependent predator-prey model with Holling type functional response, J. Comput. Appl. Math. 162 (2004), no. 2, 341-357.

Lin-Lin Wang: Depatement of Mathematics, Tianjin University, Tianjin 300072, China

Current address: Department of Mathematics, Lanzhou University, Lanzhou, Gansu 730000, China

Wan-Tong Li: Department of Mathematics, Lanzhou University, Lanzhou, Gansu 730000, China E-mail address: wtli@lzu.edu.cn

Pei-Hao Zhao: Department of Mathematics, Lanzhou University, Lanzhou, Gansu 730000, China E-mail address: zhaoph@lzu.edu.cn 


\section{Boundary Value Problems}

\section{Special Issue on}

\section{Singular Boundary Value Problems for Ordinary Differential Equations}

\section{Call for Papers}

The purpose of this special issue is to study singular boundary value problems arising in differential equations and dynamical systems. Survey articles dealing with interactions between different fields, applications, and approaches of boundary value problems and singular problems are welcome.

This Special Issue will focus on any type of singularities that appear in the study of boundary value problems. It includes:

- Theory and methods

- Mathematical Models

- Engineering applications

- Biological applications

- Medical Applications

- Finance applications

- Numerical and simulation applications

Before submission authors should carefully read over the journal's Author Guidelines, which are located at http://www.hindawi.com/journals/bvp/guidelines.html. Authors should follow the Boundary Value Problems manuscript format described at the journal site http://www .hindawi.com/journals/bvp/. Articles published in this Special Issue shall be subject to a reduced Article Processing Charge of $€ 200$ per article. Prospective authors should submit an electronic copy of their complete manuscript through the journal Manuscript Tracking System at http://mts.hindawi.com/ according to the following timetable:

\begin{tabular}{|l|l|}
\hline Manuscript Due & May 1,2009 \\
\hline First Round of Reviews & August 1, 2009 \\
\hline Publication Date & November 1,2009 \\
\hline
\end{tabular}

Compostela, Santiago de Compostela 15782, Spain; juanjose.nieto.roig@usc.es

\section{Guest Editor}

Donal O'Regan, Department of Mathematics, National University of Ireland, Galway, Ireland; donal.oregan@nuigalway.ie

\section{Lead Guest Editor}

Juan J. Nieto, Departamento de Análisis Matemático, Facultad de Matemáticas, Universidad de Santiago de 